\title{
DISPERSION COMPENSATION IN AN OPTICAL FIBER BY USING CHIRP GRATINGS
}

\author{
Parul Singh ${ }^{1}$ \\ ${ }^{I}$ M.tech Student of ECE, ECE Department, Government Women Engineering College, Rajasthan, India
}

\begin{abstract}
A fiber bragg grating $(F B G)$ is one of the most important and applicable component in an optical communication system. In this paper, the use of chirped FBG has been studied as a dispersion compensator in an optical communication system. The simulation model of the chirp grating based on the optisystem 7.0 is presented according to the above principle. The simulation results are validated by analyzing the $Q$-factor, we examined the effect of this component in the data receiver.
\end{abstract}

Keywords: Fiber Bragg grating (FBG), dispersion compensation and optical communication.

\section{INTRODUCTION}

Chromatic dispersion is the phenomenon in an optical fiber which occurred due to dependence of group index $(\mathrm{Ng})$ to wavelength. Dependence of $\mathrm{Ng}$ to wavelength in an optical fiber produces a time extension in propagated pulses. Extension of pulses after a distance leads to errors in receiver [2]. Using erbium doped fiber amplifiers (EDFAs) in an optical fiber communication system is an offer to compensate loses. Moreover, dispersion compensation fibers (DCFs) are extensively used to compensate chromatic dispersion. This method required to use DCFs negative dispersion coefficient in a communication link in order to disable the effect of positive dispersion in fibers. Now days, FBG are suggested to compensate chromatic dispersion in fibers. A FBG is a type of distributed Bragg reflector constructed in a small segment of an optical fiber that reflects particular wavelengths of light and transmits all other. This is achieved by producing a periodic variation in the refractive index of the fiber core. Transmitted light in an FBG core which satisfies the Bragg conditions is resonated by grating structure and reflected. A FBG can therefore be used as an optical filter to block certain wavelengths. This filter has various applications which improve the quality and reduce the cost of an optical network. The refractive index profile of the grating may be varied to add some features, such as a linear variation in the grating period, called a chirp. The reflected wavelength changes with the grating period, broadening the reflected spectrum. The most important inclination of chirp FBG than other recommended types are small internal lose and cost efficiency [4].

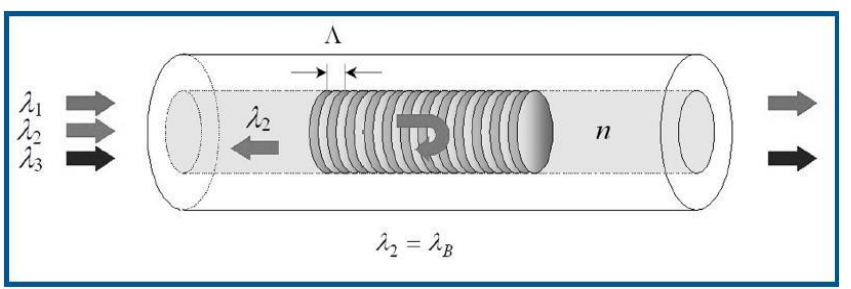

Fig -1: Principle of operation of a FBG [1].

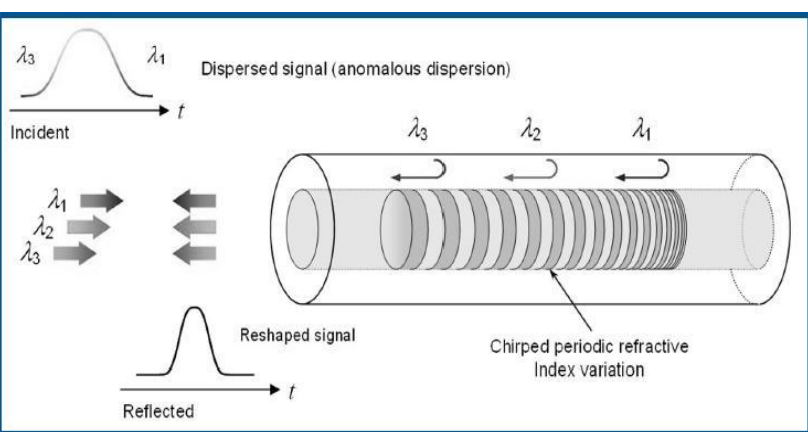

Fig - 2: A chirped FBG compensate for dispersion by reflecting different wavelengths at different locations along the grating lengths [1].

\section{FBG OPERATION PRINCIPLES}

FBG is the addition of another modulation of refractive index which acts like a wavelength selective mirror as shown in "Fig. 1,". FBGs were firstly seen as a result of strong argon ion laser radiation to a fiber with germanium dope. Later, various methods were employed in order to map grating in optical fiber in which wide-ranging types of pulsed and continuous lasers were used in visible and ultraviolet region [3]. Subsequent gratings selectively reflect transmitted light in fiber according to Bragg wavelength which is given as follow

$$
\Lambda_{\mathrm{B}}=2 \mathrm{n} \Lambda
$$

In this equation, $\mathrm{n}$ and $\Lambda$ are refractive index of core and grating period in fiber, respectively. A uniform grating can be expressed as sinusoidal modulation of fiber core refractive index:

$$
\mathrm{n}(\mathrm{z})=\mathrm{n}_{\mathrm{core}}+\delta \mathrm{n}[1+\cos (2 \mathrm{z \Pi} / \Lambda+\varphi(\mathrm{z}))]
$$

In which $\mathrm{n}_{\text {core }}$ is the core refractive index when it is not radiated and $\delta n$ is amplitude of induced refractive index variations. 


\subsection{Chirp FBGs and Dispersion Compensate}

A chirp is where variations in grating period are formed along the grating. As shown in "Fig 2," when a signal arrives into chirp, different wavelengths are reflected from different parts of grating. Thus, a delay subjected to wavelength of signal is created by grating. Some wavelengths have more expansion than others. This feature is used for dispersion compensating in communication links.

\section{SIMULATION OF TRANSMISSION SYSTEM}

We use the parameters in Table 1 in mandate to simulate the system. The model of simulated system is as shown in "Fig 3 ,". In this simulation, we apply a continuous wave (CW) laser with frequency of $193.1 \mathrm{THz}$ and output power of 1 $\mathrm{mW}$ which is externally modulated at $10 \mathrm{Gbits} / \mathrm{s}$ with a nonreturn to zero (NRZ) pseudorandom binary sequence in a Mach-Zehnder modulator with $30 \mathrm{~dB}$ of extinction ratio. Employed EDFA in this model has the gain amount of 20 $\mathrm{dB}$ and noise figure of $4 \mathrm{~dB}$ which is not depends on wavelength and ignorable noise that is only used for dispersion compensating and non-linear effects in transmission system. "Fig 4," shows a eye diagram of reflected spectra of FBG after dispersion compensation. FBGs have following parameters as shown in Table 2.

Table -1: Fiber parameters

\begin{tabular}{|l|l|}
\hline Parameters & Values \\
\hline Dispersion $(\mathrm{ps} / \mathrm{km} / \mathrm{nm})$ & 16.75 \\
\hline $\begin{array}{l}\text { Dispersion } \\
\text { slope }\left(\mathrm{ps} / \mathrm{nm}^{2} / \mathrm{km}\right)\end{array}$ & 0.075 \\
\hline $\begin{array}{l}\text { Attenuation } \\
\text { index }(\mathrm{dB} / \mathrm{km})\end{array}$ & 0.20 \\
\hline Length of fiber $(\mathrm{km})$ & 10 \\
\hline
\end{tabular}

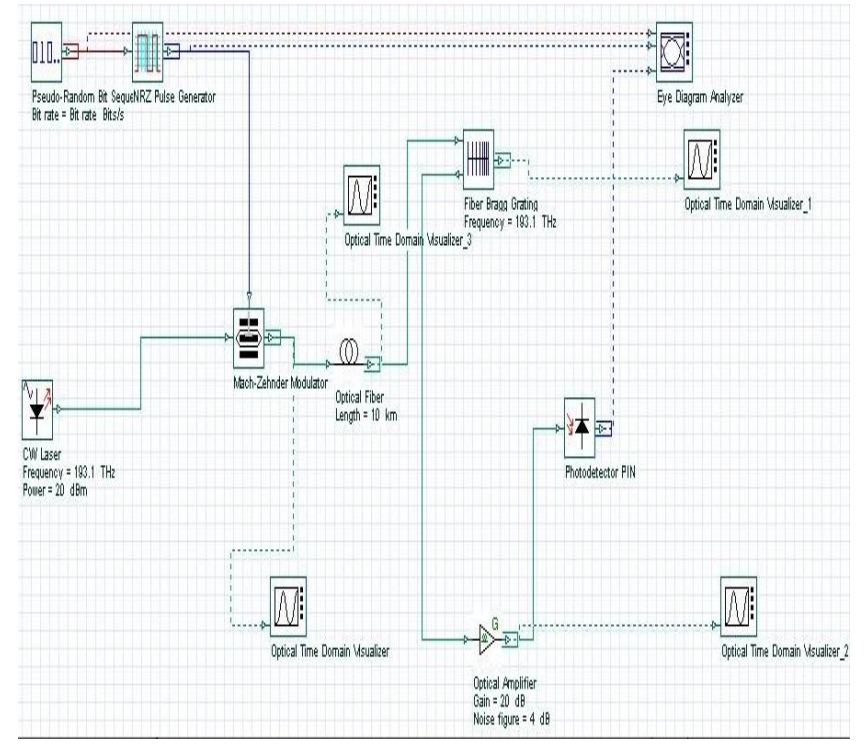

Fig -3: A simulated system with optisystem software

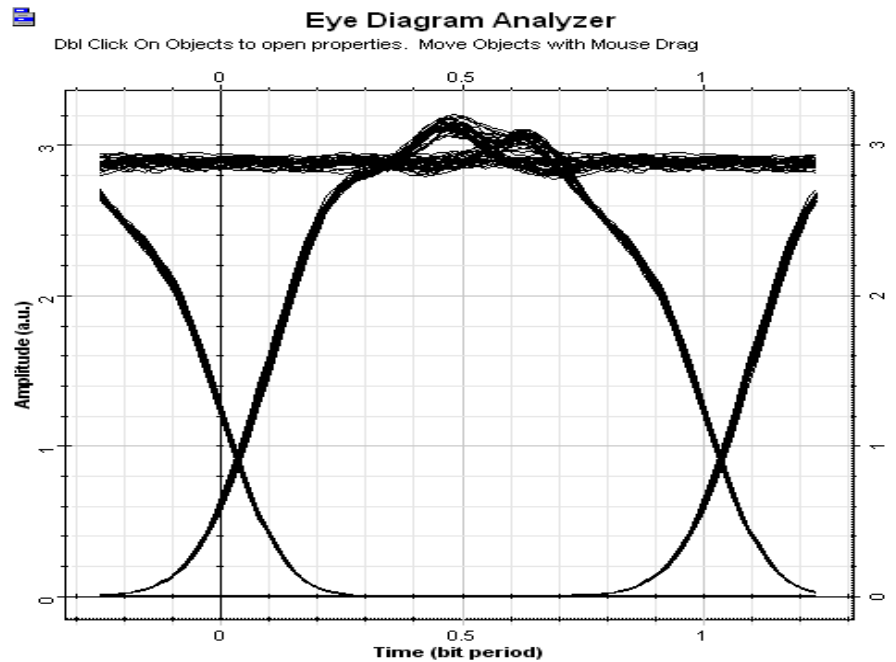

Fig -4: The eye diagram of a signal reflected from FBG

Table -2: Fbg parameters

\begin{tabular}{|l|l|}
\hline Parameters & Values \\
\hline Frequency(THz) & 193.1 \\
\hline Effective refractive index & 1.45 \\
\hline Length of grating(mm) & 6 \\
\hline Apodization function & Tanh \\
\hline Tanh parameter & 4 \\
\hline Chirp function & Linear \\
\hline Linear parameter $(\mu \mathrm{m})$ & 0.0001 \\
\hline
\end{tabular}

We attained the most proper length for projected model equal to $1=6 \mathrm{~mm}$. As shown in "Fig. 5," the eye diagrams of different profiles of apodization illustrated that Tanh is the most suitable among them.

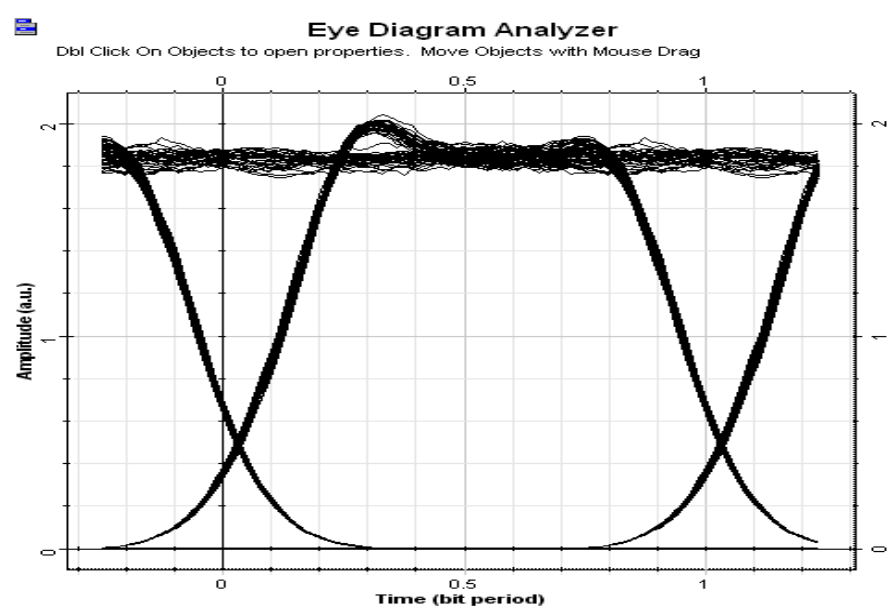

(a) 


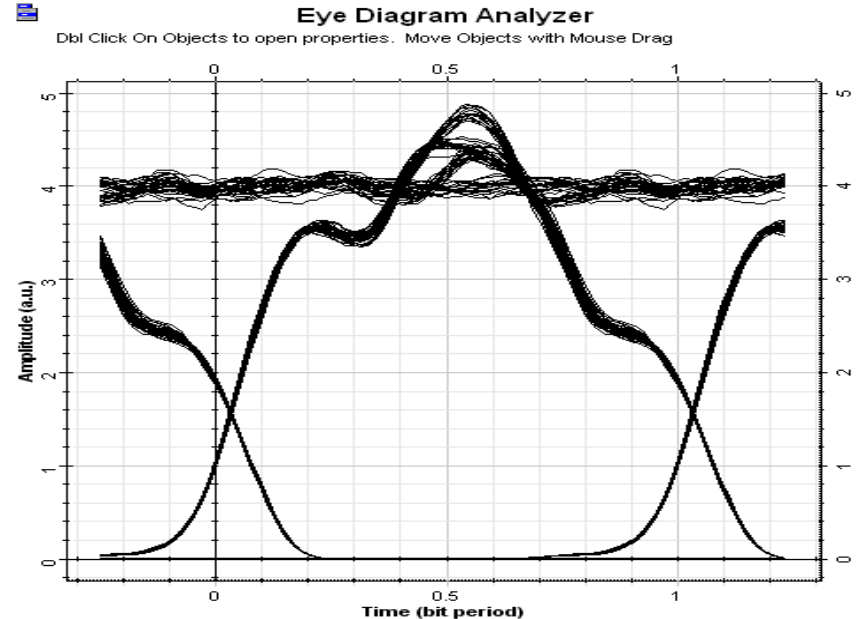

(b)

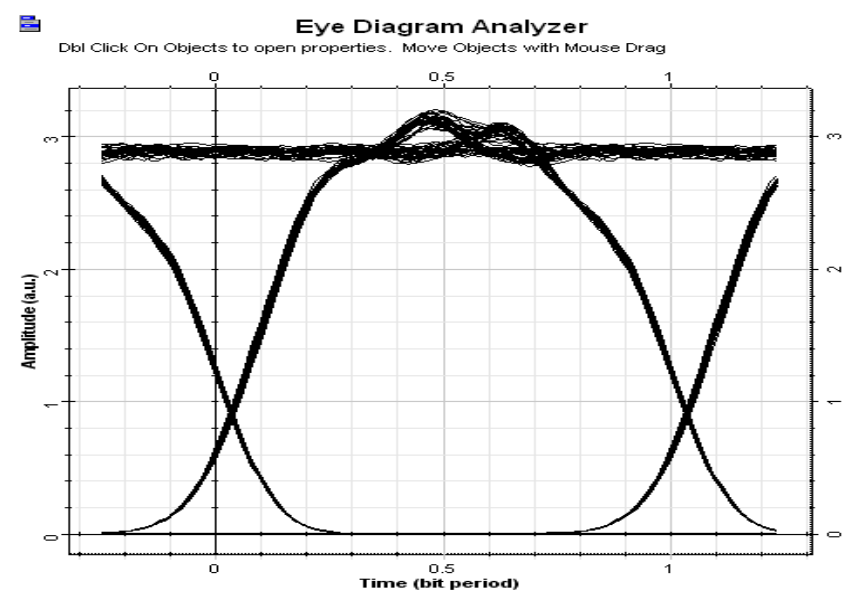

(c)

Fig -5: Eye diagram from different apodization function: (a) Gaussian function (b) Uniform function and (c) Tanh function

Here we discover that reducing the chirp parameter toward the ideal amount (0.0001) is adjustable for getting the best output.

\section{CONCLUSIONS}

In this paper, we simulated a optical communication system in an information transmission. As soon as we saw dispersion, we decided to compensate it in mandate to receive data at receiver. For this purpose, we used chirp FBG and simulated it. Also, it can be obtained that increase in grating length leads to decrease in pulse extension, and also increase in power. By seeing the power of the output spectrum of modulator and the pulse shape in that point, the most appropriate length which equals to $6 \mathrm{~mm}$ can be followed. Apodization function is not very effective in FBG reflected spectrum, although the favourable shape is Tanh function due to its grating length. Lastly, it can be understood that the pulse was broadened and its power is increased as a result of the increase in the chirp parameter which is the best volume.

\section{ACKNOWLEDGEMENTS}

The author is thankful to everyone who supported or motivated me.

\section{REFERENCES}

[1] S. O. Mohammadi, Saeed Mozaffari, and M. Mehdi Shahidi, "Simulation of a transmission system to compensate dispersion in an optical fiber by chirp gratings" International journal of the Physical Sciences vol. 6(32), pp. 7354-7360, 2 Dec 2011.

[2] Luis M, Rui, "Characterization of fiber Bragg grating for dispersion compensation," Thesis of postgraduate, pp. 4-18, 2004.

[3] Raman. K, "Fiber Bragg gratings," 3rd edition, Academic Press, 1999.

[4] Isa N, Ahmet A, "Design of a chirped fiber Bragg grating for use in wideband dispersion compensation," The fourth International Conference on Electrical and Electronics Engineering ELECO, pp. 114-123, 2005.

[5] O. A, K. K, "Fiber Bragg Grating: Fundamentals and Application in telecommunication and Sensing," Artech House, Boston, pp. 189-269, 1999.

[6] M. D, "In Theory of Dielectric Optical Waveguides," Academic Press, New York.

\section{BIOGRAPHIE}

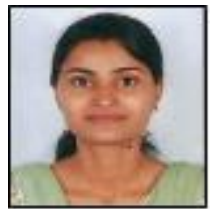

Parul Singh is born in 1989 at Ajmer of Rajasthan, India. She is pursuing M.Tech $2^{\text {nd }}$ year in Digital Communication from Rajasthan Technical University. E-mail: parulsingh.ece@gmail.com 